\title{
Modeling of Re-Sputtering Induced Bridge of Tungsten Bit-Lines for NAND Flash Memory Cell with 37nm Node Technology
}

\author{
Byungjoon Hwang, Yero Lee ${ }^{1}$, Jeong-Guk Min ${ }^{1}$, Hwakyung Shin, Namsu Lim, \\ Sungjin Kim ${ }^{1}$, Won-Young Chung ${ }^{1}$, Tai-Kyung Kim ${ }^{1}$, Jang-Ho Park, \\ Yun-Kyoung Lee, Donghwa Kwak, Jaekwan Park and Won-Seong Lee \\ Advanced Technology Development Team, CAE Team ${ }^{1}$ \\ Semiconductor R\&D Center, Memory Business, Samsung Electronics Co., Ltd. \\ San \#24, Nongseo-Dong, Kiheung-Gu, Yongin-City, Kyungki-Do, Korea, 449-711 \\ fuzzycom.hwang@samsung.com
}

\begin{abstract}
As the design rule is scaled down, the electrical isolation of metal lines becomes critical. In a high density flash memory with $37 \mathrm{~nm}$ (pitch=74nm) technology, the threshold voltage shift of $\sim 0.3 \mathrm{~V}$ is found to be caused by tungsten micro-bridge between adjacent bit-lines. Simulations and experimental data showed that tungsten re-sputtering is occurred during the deposition of HDP (High Density Plasma)- $\mathrm{SiO}_{2}$ used as the filling dielectric between tungsten bit-lines. In this paper, the model for the tungsten re-sputtering is presented. The plasma simulations are performed to investigate the effects of process factors of $\mathrm{HDP}-\mathrm{SiO}_{2}$ deposition on the formation of micro-bridge using in-house tool, PIE simulator.
\end{abstract}

\section{Introduction}

The threshold voltage distribution of about $1 \mathrm{~V}$ or less should be obtained after programming in the MLC (Multi level Cell) flash devices with 37nm technology [1][2]. Recently, a few random bits, whose threshold voltages are about $0.3 \mathrm{~V}$ higher

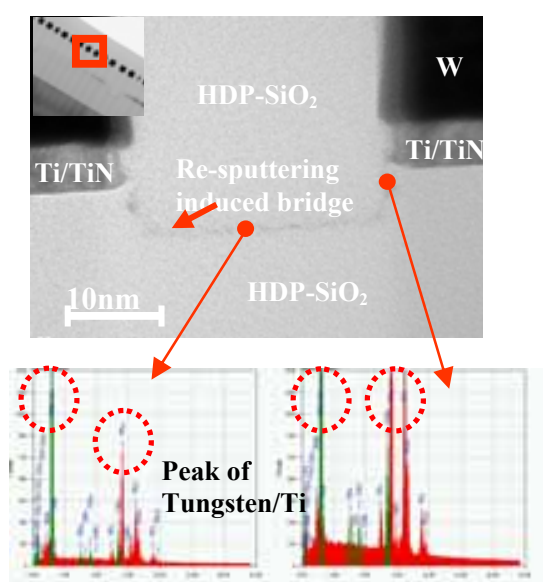

Figure 1: TEM image of tungsten bit-lines after deposition of HDP-SiO2, and the spectrum analysis of re-sputtering induced bridge

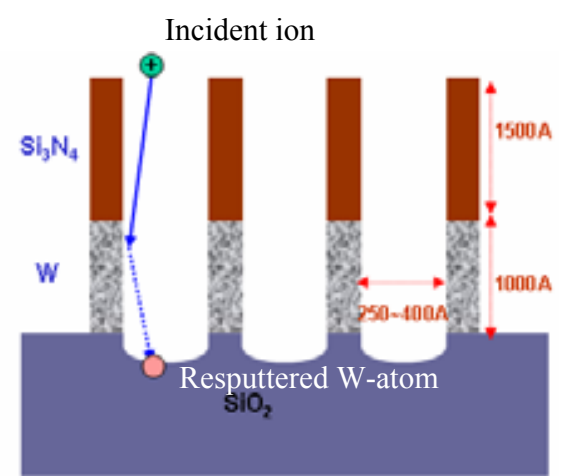

Figure 2: Structure of bit-lines with $37 \mathrm{~nm}$ (pitch $=74 \mathrm{~nm}$ ) and tungsten re-sputtering mechanism in plasma conditions when deposition of $\mathrm{HDP}-\mathrm{SiO}_{2}$ 
than those of other cells in the array, have been found. We believe that tungsten micro-bridges between tungsten bit-lines (Fig. 1) cause the shift of cell $V_{\text {th }}$. In $37 \mathrm{~nm}$ technology, the high density plasma has been used to pattern tungsten bit-line and to deposit HDP (High Density Plasma)- $\mathrm{SiO}_{2}$ as ILD (Inter-Layer Dielectric). Incident ions from the plasma can sputter the tungsten of bit-line and the sputtered tungsten can be re-deposited on the top of etched dielectric layer between bit-lines as shown in Fig2. In order to figure out which process step is dominant in the formation of tungsten micro-bridge, the flux of sputtered tungsten is investigate for several factors such as $\mathrm{W}$ thickness, sidewall slope and height of bit-line, and bias power and pressure of equipment, etc. Simulations are performed using in-house tool, PIE simulator [3]. The global model is used in the plasma simulation and Rilley model is used to calculate the ion energy and the angle distribution. The 2D profile simulation using level set method is performed to calculate the tungsten particle distribution by ionic sputtering.

\section{Modeling}

Fig. 3 shows the overall simulation procedure to analyze the re-sputtering of tungsten from the bit-lines. After obtaining the flux of ion species, IEAD (Ion Energy Angle Distribution) and sputtering yield function is obtained by the plasma simulation and

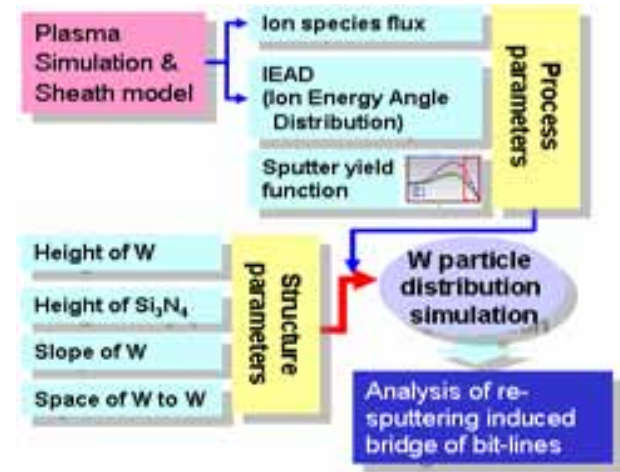

Figure 3: the sequence of simulation to analyze the bridge mechanism using in-house tool, PIE simulator

sheath model, the tungsten particle distribution are simulated for different structure parameters. The angular distribution of incident ion and sputtering yield function are obtained for each plasma conditions.

\section{Results and Discussion}

Fig. 4 shows the two different angles $(\alpha, \beta)$ of incident ion. The angular probability of incident ions is maximized at $\beta=90^{\circ}$ as shown in Fig.5. But, sputtering yield has maximum value at $\alpha=55^{\circ}$ and then decreases with angle $\alpha$ (Fig.6). Fig. 7 represents the process conditions for bit line etching and $\mathrm{HDP}-\mathrm{SiO}_{2}$ deposition, and the structural dimensions of bit-line. The flux of re-sputtered tungsten increases as the space 


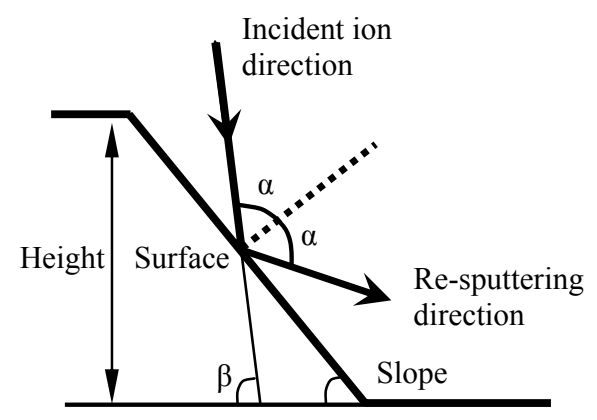

Figure 4: Incident ions sputter the surface of tungsten and re-deposit on the bottom between bit-lines

between bit-lines becomes narrower (Fig.8). The amount of re-deposition at the edge of space is more than that of the center. Fig. 9 shows how the flux of re-sputtered tungsten changes by change of source power, bias power, gases and pressure during the bit line formation process proceeds. The flux increases as the source power and pressure increase. Similar results are also obtained in $\mathrm{HDP}_{-} \mathrm{SiO}_{2}$ deposition (Fig. 10). However, the amount of re-sputtered tungsten flux in $\mathrm{HDP}-\mathrm{SiO}_{2}$ deposition is more than 10 times that of etching process. The dependences on the structural parameters

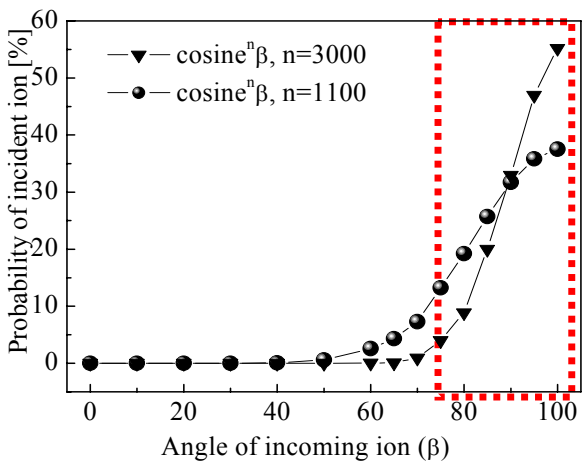

Figure 5: Probability of incident ions for angle of incident ion $(\beta)$ according to directionality

\begin{tabular}{|c|c|c|c|c|}
\hline $\begin{array}{c}\mathrm{HDP} \\
\mathrm{SiO}_{2}\end{array}$ & \multicolumn{3}{|c|}{$5000 \mathrm{~W} / 1800 \mathrm{~Wb} / * \mathrm{AR}^{*} \mathrm{SiH}_{4} / 5 \mathrm{mT}$} \\
\hline $\mathrm{Etch}$ & \multicolumn{4}{|c|}{$500 \mathrm{~W} / 150 \mathrm{~V} / * \mathrm{CL}_{2} / * \mathrm{~N}_{2} / * \mathrm{AR} / 4 \mathrm{mT}$} \\
\hline $\begin{array}{c}\text { Size } \\
\text { of } \\
\mathrm{W}\end{array}$ & $\begin{array}{c}\text { Height } \\
\text { of } \\
\mathrm{W}\end{array}$ & $\begin{array}{c}\text { Height } \\
\text { of } \\
\mathrm{S}_{1} \mathrm{~N}_{4}\end{array}$ & $\begin{array}{c}\text { Slope } \\
\text { of } \\
\mathrm{W}\end{array}$ & $\begin{array}{c}\text { Space } \\
\text { of } \\
\mathrm{W} \text { to } \mathrm{W}\end{array}$ \\
\hline $370 \AA$ & $1000 \AA$ & $1500 \AA$ & $85.6^{\circ}$ & $400 \AA$ \\
\hline
\end{tabular}

Figure 7: Process conditions of etching and $\mathrm{HDP}-\mathrm{SiO}_{2}$ equipment, and dimension of bit-line

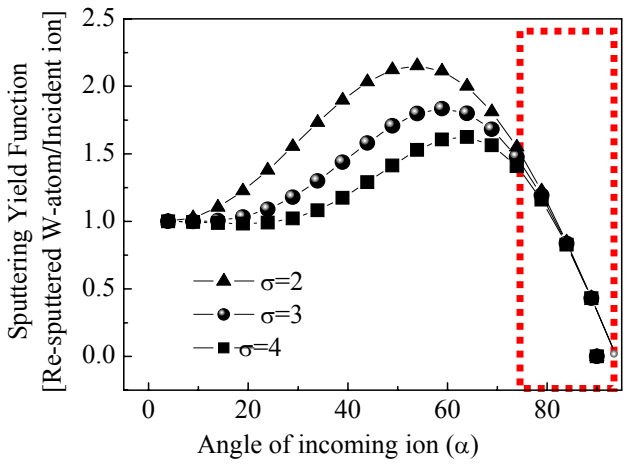

Figure 6: Sputtering yield function for angle of incident ion $(\alpha)$ for sigma values

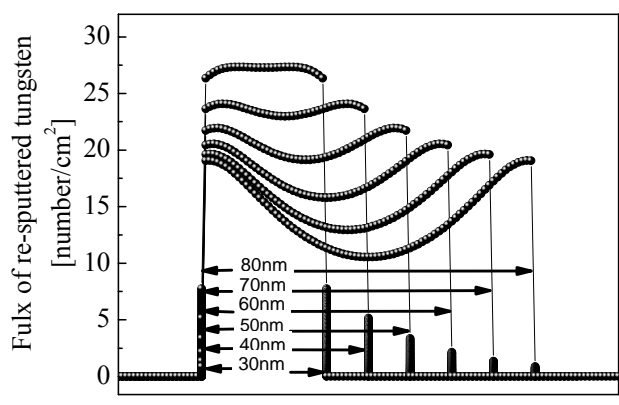

Space between tungsten bit-lines

Figure 8: Flux of re-sputtered tungsten for space size. 
are shown in Fig. 11. In order to prevent the re-sputtering of tungsten from the bitlines during $\mathrm{HDP}-\mathrm{SiO}_{2}$ deposition, the buffer $\mathrm{SiO}_{2}$ is deposited before $\mathrm{HDP}-\mathrm{SiO}_{2}$ deposition. As a result, the bridge-free bit-lines are obtained, and the random bits showing higher $\mathrm{V}_{\text {th }}$ disappear.

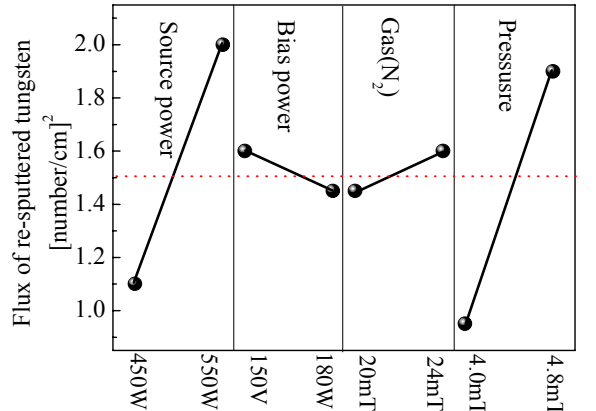

Figure 9: Flux of re-sputtered tungsten as the parameters of etching equipment are increased by $20 \%$

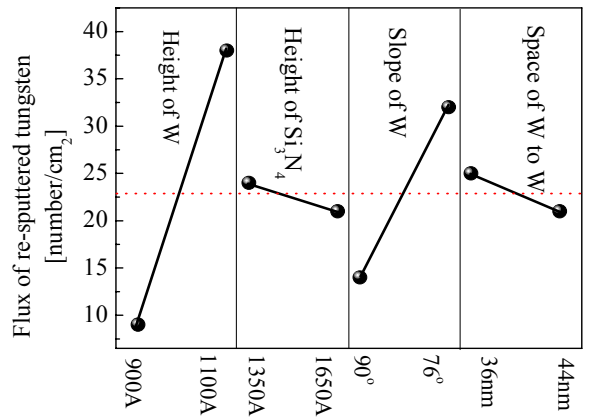

Figure 11: Flux of re-sputtered tungsten as the parameters of bit-line dimension are increased by $20 \%$

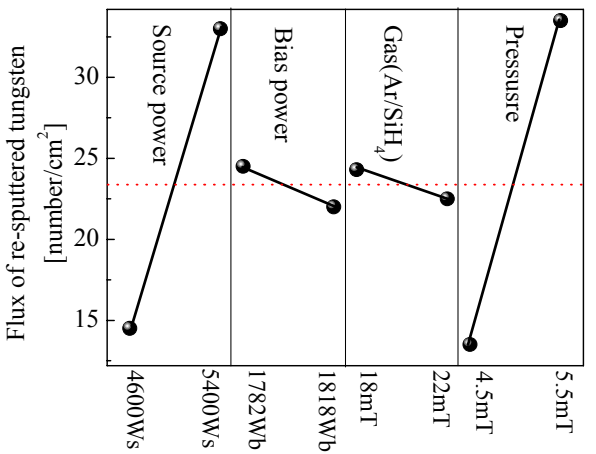

Figure 10: Flux of re-sputtered tungsten as the parameters of $\mathrm{HDP}-\mathrm{SiO}_{2}$ equipment are increased by $20 \%$

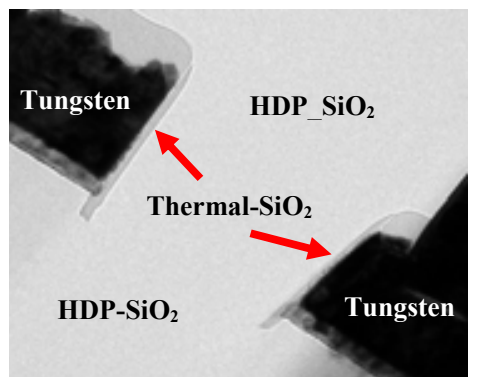

Figure 12: TEM image of bridge-free bit-lines by using thermal- $\mathrm{SiO}_{2}$ as a buffer layer before deposition of $\mathrm{HDP}-\mathrm{SiO}_{2}$

\section{Conclusion}

The tungsten re-sputtering from the bit lines under the high density plasma is investigated using in-house tool, PIE simulator, for a high density flash memory with $74 \mathrm{~nm}$ pitch. Re-sputtering is found to occur during $\mathrm{HDP}_{-} \mathrm{SiO}_{2}$ deposition. By introducing a new buffer oxide before $\mathrm{HDP}-\mathrm{SiO}_{2}$ deposition, bridge-free bit-lines are obtained (Fig. 12).

\section{References}

[1] Kinam Kim et al., "Future Outlook of NAND Flash Technology for 40nm Node and Beyond", IEEE NVSMW, pp. 9 11, 2006.

[2] Jung-Dal Choi et al., "A 0.15 um NAND Flash Technology with 0.11 um2 Cell Size for 1 Gbit Flash Memory”, IEDM, pp. 767 770, 2000

[3] Won-Young Chung et al., "Integrated Simulation of Equipment and Topography for Plasma Etching in the DRM Reactor", SISPAD, pp. 127 130, 2000. 